tungen derselben dahin berichtigen, dass selbst schon die ganz frisch hergestellten alkoholischen Losungen niemals Chlorophyll, sondern stets Chlorophyllan enthalten: Die Lösung wird niemals reingrün, sondern im günstigsten Falle gelbgrün und die spectroscopische Prüfung zeigt die Chlorophyllancharaktere in ausgeprägtestem Maasse, ${ }^{1}$ mit Zinkstaub erhitzt, wird die Lösung jedoch grün.

Es ist dies, wenn wir von dem noch fraglichen Befunde bei Bonelia viridis absehen, das einzige Thier, in dem bislang ein dem Chlorophyll der Pflanzen ausserordentlich nahestehender Farbstoff, - er entsteht ja daraus einfach durch Oxydation - oder dieses selbst gefunden wurde, da wir nach Brandt's Untersuchungen, ${ }^{2}$ die grün gefärbten Infusorien wohl als symbiotisch mit Algen vergesellschaftete Wesen auffassen müssen.

Ich behalte mir vor, im nächsten Sommer auch andere grüngefärbte Raupen und Käfer (Cetonia aurata, Cerambyx moschatus und andere) auf Chlorophyll zu untersuchen.

Aus das regelmässig grüngefärbte Cajeputöl habe ich, um der immer noch von einigen Seiten aufgestellten Behauptung, dass die grüne Farbe von Chlorophyll herrühre, zu begegnen, in den Kreis meiner Beobachtungen gezogen. In den mir vorliegenden Proben fand ich jedoch niemals auch nur die geringsten Anzeichen vorhandenen Chlorophylls, die grüne Farbe ist daher unbestreitbar auf den ubrigens sehr geringen Kupfergehalt des Oels zurückzuführen. ${ }^{3}$

\title{
Bemerkungen über die botänische Nomenclatur der Pharmacopöe.
}

Von F. A. Flückiger.

Herr Geh. Rath Prof. Dr. Göppert, Director des botanischen Gartens in Breslau, veroffentlichte voriges Jahr die Schrift: "Unsere officinellen Pflanzen " als Nachtrag zu seinen frühern lehrreichen Arbeiten auf diesem Gebiete. Die 12 Seiten des vorliegenden Heftes werden in folgender Weise eingeleitet: „Bei der immer mehr schwindenden Theilnahme an officinellen Gewächsen,

1) vergl. Fig. 4 auf Seite 139.

2) Ueber das Zusammenleben von Thieren und Algen, Sitzungsber. d. Ges. naturforschender Freunde. Berlin, November 1881.

3) vergl. auch Flückiger, Pharmacognosie des Pflanzenreiches. p. 150 
F. A. Flückiger, Bemerk. üb. d. botanische Nomenclatur d. Pharmacopöe. 147

halte ich es im Interesse der systematischen Botanik und der heutigen Pharmacie fast für geboten, durch eine Zusammenstellung aller etwa bis 1870 in der medicinisch-pharmaceutischen Welt noch bekannteren Arten und der von ihnen stammenden Droguen, viele dieser heut nur mehr oder weniger beachteten Pflanzen der Vergessenheit zu entreissen und hier möglichst genau aufzuführen. Zur Hebung dieses Interesses wird jedenfalls nicht beigetragen durch das Verfahren der neuen Reichspharmacopøe, die Autoren der in ihr aufgeführten Arten wegzulassen, ein nicht zu billigendes Vorgehen, welches in weitem Maasse Unsicherheit darüber herrorruft, welche Art denn eigentlich gemeint sei, wie z. B.: (Die Nummern von mir beigesetzt)"

1) Unter Alö̈ lingua gehen elf verschiedene Arten! - Es giebt eine Aloë vulgaris von $\mathrm{DeCa}_{\theta} \mathrm{C}$ dolle, eine andere von Willdenow eine dritte von Lamarck.

2) Copaifera officinalis ist ohne Autorangabe ein Sammelname, der eine ganze Anzahl Arten und Formen umfasst.

3) Unter Eugenia caryophyllata sind 2 weit von einander entfernte Pflanzen, die eine von Thun berg, die andere von Swartz, beschrieben worden. Von der Thunberg'schen Art stammen die Gewürznelken, während die Swartz'sche Art (Syzygium Jambolanum DC.) für die Pharmacie ohne Bedeutung ist.

4) Malva vulgaris ist je nach dem Autor Malva silvestris L., M. rotundifolia L., M. neglecta Wallr., oder umfasst mehrere dieser Arten gemeinsam.

5) Unter Mentha crispa haben Linné, Roth und Tenore 3 verschiedene Arten gemeint.

6) Pinus australis ist je nach dem Autor eine afrikanische, mediterrane oder nordamerikanische Fichtenart.

7) Melilotus officinal is ohne Autorenangabe umfasst so ziemlich alle mitteleuropäischen gelbblühenden Meliloten. Melilotus altissima ron Schultes ist die weissblühende $\mathbf{M}$. alba Desr., M. altissima Thuill. die gelbbluhende Art.

8) Quercus Robur; 2 Arten, pedunculata Willdenow und Robur Willd.

9) Unter Lactuca virosa verstanden Linne, Hablizl und Thunberg 3 sich sehr fern stehende Species.

10) Von Verbascum phlomoïdes existirt eine Linné'sche und eine Sprengel'sche Art. 
148 F. A. Flïckiger, Bemerk. üb. d. botanische Nomenclatur d. Pharmacopöe.

11) Ononis spinosa Linné's ist etwas ganz anderes als 0 . spinosa Hasselquist."

Es ist allerdings üblich, in jeder botanischen Arbeit, welche auch nur einigermaassen au Wissenschaftlichkeit Anspruch erhebt, die Pflanzen mit den Namen der Autoren auszustatten. Wird dazu auch noch das Werk angegeben, in welchem der angeführte Schriftsteller die betreffende Pflanze beschrieben und abgebildet hat, oder das Herbarium, in welchem dieselbe aufbewahrt liegt, dann wird wohl meistens ein Missverständniss ausgeschlossen sein. In dieser Weise muss namentlich derjenige verfahren, welcher sich die kritische, systematische Durcharbeitung einer Pflanzengruppe oder irgend einer grössern Abtheilung des Pflanzenreiches zur Aufgabe macht. Unbedingt muss dieses Verfahren auch da verlangt werden, wo es sich um weniger allgemein bekannte Pflanzen handelt. So weit und noch ein Stück weiter bin ich einverstanden mit dem Gebrauche, die botanischen Autoren anzuführen, doch kann ich mich allerdings nicht erinnern, jemals gehört oder gelesen zu haben, dass z. B. in irgend einem Examen vom Candidaten die Kenntniss solcher "Autornamen " verlangt worden wäre oder dass davon in Vorlesungen über Botanik viel die Rede sei, sofern es sich nicht um ganz specielle Systematik handelt.

Meines Erachtens durfte die Pharmacopöe sich recht wohl auf einen eigenen Standpunkt versetzen. Sie ist kein wissenschaftliches Werk, sondern ein Gesetzbuch, welches allerdings, wie auch andere Gesetzbücher, auf wissenschaftlichem Boden steht. Sie macht die Drogen namhaft, welche der Apotheker dem Mediciner zur Verfügung halten soll, und schildert dieselben so weit, als es zur Beurtheilung der Identität erforderlich ist. Mehr will und soll die Pharmacopöe im wesentlichen nicht leisten. Man könnte sogar behaupten, dass sie im Unrecht ist, wenn sie Stammpflanzen nennt, welche den deutschen Apothekern und Aerzten so gut wie niemals zu Gesichte kommen, d. h. wenigstens nicht in ihrem Berufsleben. Indem die Pharmacopöe Aloë, Ammoniacum, Asa foetida, Balsamum Copairao, Balsamum peruvianum, Benzoë u. s. w. kurz und möglichst zutreffend schildert, erfüllt sie ihre Aufgabe, thut aber ein übriges, wenn sie auch noch die Pflanzen erwähnt, von welchen die eben genannten Drogen abstammen. Wie viele Leser der Pharmacopöe sind in der Lage, sich von der Richtigkeit jener botanischen Angaben des Buches zu überzeugen? Es ist nur ein Stück Belehrung, 
welches die Pharmacopöe hier bietet, so dass sie eigentlich schon aus ihrer Rolle fällt und zur Lehrerin wird, wo sie nur befehlen soll. Anders liegen die Dinge doch wohl, wenn es sich um Amygdalae, Cortex Frangulae, Cortex Quercus, Folia Althaeae, Folia Uvae ursi, Radix Taraxaci, Tubera Aconiti u. s. w. handelt. Hier ist der Apotheker moglicherweise doch in der Lage, sich wirklich an die von der Pharmacopðe vorgeschriebene Pflanze, halten zu können; die genaue Bekanntschaft mit der letzteren wird ihm von Nutzen sein und von ihm verlangt werden dürfen.

Es handelt sich also im Grunde um 2 Kategorien von Pflanzen, einerseits nämlich einfach um inländische oder doch leicht zugängliche und anderseits um exotische, eine Unterscheidung, welche bei der Erörterung der von Herrn Geh. Rath Gojpert aufgeworfenen Frage auch nicht ganz übersehen werden darf. Zu Gunsten des von der Pharmacopöe eingeschlagenen Verfahrens habe ich mich schon einmal in der Beilage zur I'harm. Zeitung vom 26. Februar 1881 geäussert und erlaube mir, darauf zu verweisen, um Wiederholungen zu vermeiden. Grundsätzlich habe ich in der That wenig beizufïgen; wenn sich aber ein so hoch verdienter und so gründlich orientirter Gönner und Forderer der Pharmacie, wie Herr Geh. Rath Göppert, in gegentheiligem Sinne vernehmen lässt, so ist es Pflioht, eine solche gewichtige Stimme nicht zu überhören und zwar um 80 mehr, als er keineswegs allein steht.

Herr Geh. R. Göppert rügt zunächst, dass die Pharmacopðe durch ihr Verfahren nicht zur Hebung des botanischen Interesses an den officinellen Pflanzen beitrage. So richtig diese Bemerkung auch ist, so wenig belangreich scheint sie mir an dieser Stelle zu sein. Der Pharmacopöe liegt jene allerdings sehr wünschenswerthe Hebung eines freilich sehr schönen Interesses sicherlich nicht ob und ich glaube auch, dass für dasselbe in der neuesten einschlagenden Literatur Deutschlands anderweitig recht viel geleistet worden ist, so dass es kaum von viel Gewicht wäre, wenn die Pharmacopöe auch noch direct dazu beitrüge.

Feiner bedauert Herr Geh. Rath Göppert, dass dieselbe in weitem Maasse Unsicherheit darüber hervorrufe, welche Art im gegebenen Falle gemeint sei, und führt die oben aufgezählten 11 Beispiele an. Besehen wir uns diese näher, so ergiebt sich Folgendes.

Zu 1: In Betreff der Aloë drückt sich die Pharmacopőe mit Recht wenig bestimmt aus und legt mehr darauf Gewicht, die 
Waare zu schildern, als ihre Abstammung fest zu setzen. Einmal nimmt der Hottentotte, wenn die Noth ihn gelegentlich $z u$ diesem Erwerbe treibt, die erste beste Aloë in Arbeit, so dass niemand in Betreff der Species genau unterrichtet ist. Dann aber ist sogar zu bemerken, dass im Sinne der neuesten Bearbeitung des Genus Aloë die beiden Arten A. vulgaris und A. lingua nicht mehr genannt werden müssten. Leider aber legte Baker (Journal of the Linnean Society. Botany. Vol. XVIII. p. 148) seine sichtende Hand zu spät an das entsetzliche Chaos der Benennungen der Aloë-Arten, als dass seine Arbeit noch für die Pharmacopoea Germanica hätte benutzt werden können. Um übrigens auf A. lingua (jetzt Gasteria disticha Haworth) zurückzukommen, so liegt es für den Kritiker doch gewiss am nächsten, Thunberg's Benennung zu berücksichtigen, da er es war, der zuerst über die jetzt allein officinelle Cap-Aloë berichtete. Und die zu verschiedenen Zeiten aufgestellte Aloë vulgaris dürfte schliesslich nahe zusammenfallen. Alle diese Aloë-Arten des Caplandes geben, wie es scheint, eine und dieselbe Waare und damit müssen wir uns wohl beruhigen; niemand ist zur Zeit zu genaueren Nachweisen im Stande.

Zu 2: Es ist mir nicht bekannt, dass Copaifera officinalis nur ein Sammelname sei; ich meine, dass übereinstimmend darunter die Linné'sche Art (C. Jacquini Desfontaines) verstanden wird, auch giebt es eine Anzahl viel verbreiteter Abbildungen, z. B. hei Hayne und in der Düsseldorfer Sammlung, welche gerade diese Art darstellen. Es will mir also durchaus nicht einleuchten, dass die Pharmacopöe durch Nennung der C. officinalis ohne den Autor zu Zweifeln Anlass gebe. Vielleicht wäre es noch besser gerechtfertigt, nach den Boweisen zu fragen, welche dafür vorliegen, dass Balsam von Copaifera officinalis gesammelt werde!

Zu 3: Welche Eugenia caryophyllata die Gewürznelken liefert, ist für keinen Botaniker zweifelhaft; dass einmal von Swartz versucht worden ist, denselben Namen dem vormaligen Syzygium jambolanum DC. beizulegen, hat doch kaum noch irgend welches Interesse. Dieser letztere Baum ist in Europa äusserst wenig bekannt und ohne alle practische Bedeutung, während der Gewürznelkenbaum in allen betreffenden Kreisen hinlänglich gekannt ist; wo wäre da eine Unsicherheit denkbar, wenn die Pharmacopöe sich erlaubt, den Namen Thunberg wegzulassen! Allerdings ist wohl der Name Caryophyllus aromaticus oder aromatica geläufiger, aber eine irgendwie 
umfassendere Berücksichtigung der Synonymen liegt doch erst recht ausserhalb der Aufgabe der Pharmacopöe. In dieser Hinsicht durfte sie sich gewiss mit Recht an diejenigen Bezeichnungen halten, welche gegenwärtig in den besten einschlägigen Werken angenommen, d.h. an die Spitze gestellt sind. Diese lassen das Genus Caryophyllus nicht gelten.

Zu 4: Es ist zuzugeben, dass die hier vorzugsweise gemeinte Malva schon 1824 durch Wallroth als M. neglecta und erst 1828 durch Fries als M. vulgaris bezeichnet worden ist. Aber gerade der Umstand, dass der letztere Name nicht ausschliesslich nur die von Wallroth gemeinte Form bedeutet, mag zur Entschuldigung dienen, dass die Pharmacopöe hier gegen das Prioritätsrecht gesündigt hat. Practisch gesprochen ist es ja ganz und gar gleichgültig, von welcher der einheimischen Formen der Kräutersammler seine Malvenblätter nimmt und der Apotheker wird auch kaum in die Lage kommen, sich zu überzeugen, ob er M. neglecta Wallr., M. rotundifolia Linné's oder anderer Botaniker vor sich hat, da ja sogar die viel stärker abweichenden Blätter der $\mathrm{M}$. silvestris zulässig sind.

$\mathrm{Zu} \mathrm{5:} \mathrm{Bei} \mathrm{Mentha} \mathrm{crispa} \mathrm{ist} \mathrm{das} \mathrm{Interesse} \mathrm{der} \mathrm{Pharmacopøe}$ darauf beschränkt, eine Minze vorzuschreiben, welche den so höchst eigenthümlichen Krauseminzgeruch besitzt. Indem sie keinen Autornamen nennt, geht die Pharmacopöe den Streite über die Herkunft einer solchen Mentha aus dem Wege; sie überlüsst ganz und gar den Botanikern oder Pharmacognosten die Erörterung der Frage, ob die Krauseminze nur eine Culturform oder eine "gute Species" sei, was allerdings nicht ohne Interesse ist. Der Gleichförmigkeit der Droge in äusserlicher Hinsicht trägt die Pharmacopöe noch so weit Rücksicht, als sie Mentha viridis mit flacher, nicht krauser, Blattspreite ausschliesst; alles übrige, was sich in systematischer Hinsicht an den Namen Mentha crispa knüpft, kann und soll der Pharmacopöe gleichgültig sein, sobald ihre Krauseminze das richtige Carvol enthält.

Zu 6: Obgleich 3 Botaniker der Reihe nach 3 verschiedene Fichten mit dem Namen Pinus australis belegt haben, so ist doch sicherlich kein auch nur halbwegs Kundiger darüber im Zweifel, dass heute darunter einzig die nordamerikanische, von Michaux aufgestellte Art gemeint ist. Diesen Bescheid finden weniger gut unterrichtete Pharmaceuten ausnahmslos auch in allen Werken, an welche sie sich zu diesem Behufe etwa wenden mögen. Schon vor 
50 Jahren kannte z. B. die damals ausgezeichnete und sogar heute noch oft brauchbare medicinisch-pharmaceutische Flora von Kosteletzky nur jene eine Pinus australis. Also auch hier keine Unsicherheit!

$\mathrm{Zu} \mathrm{7:} \mathrm{Wenn} \mathrm{gegen} \mathrm{Melilotus} \mathrm{officinalis} \mathrm{die} \mathrm{Einwendung}$ erhoben wird, dass diese Angabe, ohne Autornamen, so ziemlich alle mitteleuropäischen gelbblühenden Meliloten umfasse, so darf bemerkt werden, dass es sich eigentlich wohl nur um deutsche derartige Pflanzen handelt. Und solcher giebt es im ganzen - zwei, nämlich M. officinalis Desrousseaux und M. altissimus Thuillier, also genau die von der Pharmacopöe vorgeschriebenen Stammpflanzen ihrer Herba Meliloti. Dass Schultes unter Melilotus altissimus eine dritte, nämlich eine weissblühende Art verstanden wissen wollte, kann für uns nicht in Betracht kommen, denn die Pharmacopöe fordert ausdrücklich gelbe Blüthen und kräftigen Wohlgeruch. Damit ist M. altissimus Schultes (Synonym: M. alba Desrousseaux) bestimmt ausgeschlossen und mit rollem Rechte jede aromatische Form mit gelber Blüthe zugelassen, sei sie nun von den Systematikern so oder so getauft worden.

Zu 8: Die Berechtigung der Frage nach der Bedeutung der laconischen $Q$ uercus Robur der Pharmacopöe ist einzuräumen. Ist $Q$. Robur im ursprünglichen Sinne von Linné, d. h. nur als Stieleiche, gemeint oder ist $Q$. Robur hier in der umfassendern Bedeutung mit DeCandolle als alle deutschen Eichen einschliessend gebraucht? Darüber giebt die Pharmacopöe freilich keine Auskunft; wenn man aber die höchst untergeordnete Rolle der Eichenrinde in der Pharmacio bedenkt, so kann davon keine Rede sein, dass das pharmaceutische Gesetzbuch irgend einen Grund gehabt hätte, dio Rinde einer bestimmten Form der in Dentschland wachsenden Eichen auszuschliessen und eine andere zu bevorzugen. Auch ist niemand im Stande, die Rinden von Quercus pedunculata Ehrhart und Q. sessiliflora Smith in den von der Pharmacopöe verlangten Altersstufen zu unterscheiden und wäre dieses auch möglich, so bliebe es practisch ohne allen Belang; der Gerbsäuregehalt hängt ohne Zweifel nicht von der äussern Gestalt der Eiche ab. Meines Erachtens spricht bei nur einiger Ueberlegung alles dafür, dass die Pharmacopöe unter Q. Robur alle in Deutschland wild wachsenden Formen der Eichen versteht und mit Recht verstehen darf.

Zu 9: Lactuca virosa. Wäre es möglich, dass dieser Name missverstanden werden könnte? Sollten die heutigen Systematiker 
und Pharmaceuten in der Auffassung jenes Namens nicht genau übereinstimmen? Zur Ehre der beschreibenden Botanik halte ich denselben für vollkommen sichergestellt und unzweideutig.

Zu 10: Verbascum phlomoides. Die Anforderungen der Pharmacopöe an die Wollblumen sind doch wohl bestimmt genug gefasst, um Verwechselungen und Missverständnisse auszuschliessen. Was das Sprengel'sche V. phlomoïdes betrifft, so dient mir zur Beruhigung, dass dasselbe in keinem Werke angeführt ist, welches hier irgend in Betracht kammen kann. Dasselbe fehlt sogar in der neuesten Monographie des Genus Verbascum von Franchet (angeführt in meiner Pharmacognosie, p. 746.) Um wieviel mehr werden wir also Sprengel's Verbascum phlomoïdes ignoriren dürfen!

Zu 11: Ononis spinosa. Könnte es bei einem so gemeinen Strauche möglich sein, den Namen anders zu verstehen als im Sinne Linné's und der Pharmacopöe? Auch hier darf Ononis spinosa „Hasselquist" ruhig der Vergessenheit uberlassen werden, da sie, wie es scheint, gar nicht der europäischen Flora angehört. Was in ganz Mitteleuropa überall unter 0 . spinosa verstanden wird, scheint mir ausser Zweifel zu sein.

Ich bin in den vorliegenden Bemerkungen nicht auf eine Widerlegung der von Herrn Geh. Rath Göppert erhobenen, an sich ja ganz richtigen Einwendungen ausgegangen, nur begründen meine Erörterungen, wie ich denke, den Schluss, dass jene Einwürfe gegen das Verfahren der Pharmacopöe nicht viel beweisen. Ich halte dafür, dass dieselbe sehr wohl von dem allgemeinen Gebrauche der Autorennennung abgehen durfte; im Falle der Mentha crispa (und auch der M. piperita) wäre die Angabe eines Autors sogar bedenklich gewesen, während allerdings für die übrigen Pflanzen die Beifügung der betreffenden Autoren ganz - harmlos gewesen wäre. Gerade darum konnte sie unterbleiben.

\section{B. Monatsbericht.}

Physiologische Chemie. Quelle der Hippursăure im Harn von Dr. C. Schotten. Nachdem die Untersuchungen von Salkowski, Schultzen, Meissner, Shepard, Weyl und Anrep festgestellt haben, dass im Harne, sowohl eines hungernden Menschen, als auch 